\title{
On Strong Hyperbolicity
}

\author{
By \\ Gilbert STRANG \\ (Communicated by Prof. A. Komatu, February 5, 1967)
}

1. Strongly hyperbolic systems are those which are invulnerable to perturbations of lower order; they remain hyperbolic as long as their principal part is unchanged. For constant-coefficient systems (of linear partial differential equations), hyperbolicity simply means that certain matrices $Q$ have all their eigenvalues in some half-plane $\operatorname{Re} \mu \leqslant C$. Strong hyperbolicity is then a further condition on the principal parts of these matrices. In fact, this property leads to a very neat problem in matrix analysis, which was resolved by Kasahara and Yamaguti [1, 2]; we want to discuss it further.

Let us state the problem more concretely for a first-order system

(1) $\frac{\partial u}{\partial t}=\sum_{1}^{d} A_{j} \frac{\partial u}{\partial x_{j}}+B u$.

Here $u$ is an unknown $m$-vector, $A_{j}$ and $B$ are matrix coefficients, and it is $B$ which we are allowed to change. $A$ Fourier transformation, with dual variable denoted by $\xi=\left(\xi_{1}, \cdots, \xi_{d}\right)$, yields

(2) $\frac{\partial \hat{u}}{\partial t}=\left(\sum i \xi_{j} A_{j}+B\right) \hat{u}=Q(\xi) \hat{u}$

It is on the symbol $Q(\xi)$ that one imposes the Petrowsky-Garding condition for hyperbolicity, mentioned above:

1) This work was supported by the Sloan Foundation, the National Science Foundation (NSF-GP-4364), and the Office of Naval Research. 
( 3$) \sup _{j, \xi} \operatorname{Re} \mu_{j}(Q(\xi))<\infty$.

Thus the problem is: under what conditions on the matrices $A_{j}$ does (3) hold for every $B$ ?

We shall add two alternative conditions to the one found by Kasahara and Yamaguti, and prove their equivalence. Then it is quite pleasant to show that these new conditions are necessary and sufficient for strong hyperbolicity; this simplification is really our chief contribution. The extension to arbitrary systems of Kowalevsky type is made easy by borrowing two lemmas from [2].

One of our new conditions admits a very useful interpretation: a Kowalevsky system is strongly hyperbolic if and only if it is well posed in the natural $L_{2}$ space. For the first-order system (1), this space is just $L_{2}\left(\boldsymbol{R}^{d}\right)$.

As usual, there is a parallel question, and a parallel answer, for partial difference equations. Here our results are new, and they show that an example given by Kreiss [3] is completely typical: stability which is present only in the weak (ForsytheWasow [4]) sense can always be destroyed by lower-order terms, when the eigenvalues of the principal part of the amplification matrix all have unit modulus.

We devote the Appendix to the special case of two dependent variables in the first-order system (1). It is easy to show, in any number of variables, that each of the following implies the next:

a) For some $T$, the matrices $T A_{j} T^{-1}$ are all Hermitian

b) The system (1) is strongly hyperbolic

c) Every real combination $\sum \xi_{j} A_{j}$ is similar to a real diagonal matrix.

Lax [9] has provided a counterexample to b) $\Rightarrow a$ ), and Petrowsky's counterexample [7] to c) $\Rightarrow b$ ) is reproduced in [2]. Both of these examples involve matrices of order three, and in fact it is remarked without proof in $[1,2]$ that $c) \Rightarrow b$ ) for matrices of order two. We show that actually c) $\Rightarrow a$ ) in this case; the "general case" is not reached until there are three unknowns. 
Another result of the same sort concerns the hypotheses

$\left.\mathrm{a}^{\prime}\right)$ For some $T$, the matrices $T A_{j} T^{-1}$ and $T B T^{-1}$ are all upper triangular, and the $A_{j}$ have real eigenvalues

$\mathrm{b}^{\prime}$ ) The system (1) is hyperbolic (in other words, (3) is satisfied)

Again it is trivial to show that if either a) or $a^{\prime}$ ) holds, so does $\left.b^{\prime}\right)$. For matrices of order two, we prove the converse: a hyperbolic system must satisfy a) or $\left.a^{\prime}\right)$. Inevitably, there is a counterexample of order three :

$$
\frac{\partial u}{\partial t}=\left(\begin{array}{lll}
0 & 1 & 0 \\
0 & 0 & 1 \\
0 & 0 & 0
\end{array}\right) \frac{\partial u}{\partial x}+\left(\begin{array}{rrr}
0 & 0 & 0 \\
1 & 0 & 0 \\
0 & -1 & 0
\end{array}\right) u .
$$

For this system $\mu_{j}(Q(\xi)) \equiv 0$, but the coefficient matrices cannot be made upper triangular by the same similarity [10], and the first matrix cannot be made Hermitian.

Single equations of second order are equally special.

We shall use the $l_{2}$ norm for vectors and matrices:

$$
|v|=\left(\sum\left|v_{j}\right|^{2}\right)^{1 / 2} ; \quad|A|=\sup _{|v|=1}|A v| .
$$

2. To begin, there is a familiar necessary condition :

$(H)$ : If (1) is strongly hyperbolic, then the eigenvalues $\lambda_{j}(\xi)$ of the principal part $P(\xi)=\sum i \xi_{j} A_{j}$ must be imaginary for every (real) $\xi$.

For the proof we choose $B=0$, so $Q=P$. Suppose the real part of $\lambda_{j}\left(\xi^{0}\right)$ were not zero. By homogeneity, that real part becomes arbitrarily large when we replace $\xi^{0}$ by $\beta \xi^{0}$, with $\beta$ large and of the right sign. Therefore $(3)$ is violated unless $(H)$ holds.

To see that $(H)$ is insufficient for strong hyperbolicity, we appeal to the universal counterexample

(4) $\frac{\partial u}{\partial t}=\left(\begin{array}{ll}0 & 1 \\ 0 & 0\end{array}\right) \frac{\partial u}{\partial x}+B u$.

The principal part has zero eigenvalues, so $(H)$ holds ; nevertheless

( 5 ) $B=\left(\begin{array}{rr}0 & 0 \\ -i & 0\end{array}\right) \Rightarrow Q=\left(\begin{array}{rr}0 & i \xi \\ -i & 0\end{array}\right) \Rightarrow \mu= \pm \sqrt{ } \bar{\xi}$ 
Thus the hyperbolicity of the principal part is destroyed by $B$, and (4) is not strongly hyperbolic.

Now we list the three equivalent necessary and sufficient conditions for strong hyperbolicity, of which the first appears in $[1,2]$ :

I. $(H)$ holds, and $P$ is uniformly diagonalizable: there exists $S(\xi)$ such that $S(\xi) P(\xi) S^{-1}(\xi)=D(\xi)$ is diagonal and $|S(\xi)|\left|S^{-1}(\xi)\right| \leqslant$ constant.

II. $\left|e^{t P(\xi)}\right| \leqslant$ constant, for $t \geqslant 0$ and real $\xi$.

III. $\operatorname{Re} z\left|(z-P(\xi))^{-1}\right| \leqslant$ constant, for $\operatorname{Re} z>0$ and real $\xi$. The equivalence of these three is a special case of Kreiss' important matrix theorem [5]. His theorem deals with matrices whose eigenvalues are restricted only to the left half-plane, and there is no easy proof. Here we know that $P$ has imaginary eigenvalues, and a quite simple proof is possible; we present it informally.

$$
\text { I } \Rightarrow \text { II }: \quad e^{t P}=S^{-1} S e^{t P} S^{-1} S=S^{-1} e^{t D} S .
$$

The diagonal matrix $e^{t D(\xi)}$ is unitary, by $(H)$, so that

(6) $\left|e^{t P(\xi)}\right| \leqslant\left|S^{-1}(\xi)\right||S(\xi)| \leqslant$ constant .

II $\Rightarrow$ III. Given that $\operatorname{Re} z>0$,

$$
\text { (7) } \begin{aligned}
\left|(z-P)^{-1}\right| & =\left|\int_{0}^{\infty} e^{t(P-z)} d t\right| \leqslant \text { constant } \int_{0}^{\infty} e^{-t \operatorname{Re} z} d t \\
& =\text { constant } / \operatorname{Re} z .
\end{aligned}
$$

III $\Rightarrow$ I. Given $\xi$, it is clear from III that $P(\xi)$ can have no eigenvalues in the right half-plane $\operatorname{Re} \lambda>0$. The same is true of $P(-\xi)=-P(\xi)$. Therefore the eigenvalues are imaginary, and $(H)$ holds.

Next we assert that each $P(\xi)$ is diagonalizable. If not, it is easy to check from the Jordan form that $(z-P(\xi))^{-1}$ has at least a double pole at the offending eigenvalue $\lambda$, contradicting III as $z \rightarrow \lambda$. Therefore each $P$ admits a spectral decomposition

( 8 ) $P(\xi)=\sum \lambda_{j}(\xi) E_{j}(\xi)$,

where the eigenvalues $\lambda_{j}$ are imaginary and the $E_{j}$ are mutually orthogonal projections: 
(9) $E_{j} E_{k}=\delta_{j k} E_{k}, \sum E_{j}=I$.

As suggested by [6], let

(10) $H(\xi)=m \sum E_{j}^{*}(\xi) E_{j}(\xi)$,

recalling that $m$ is the order of the matrices. Applying (9),

(11) $H(\xi) P(\xi)=-P^{*}(\xi) H(\xi)$.

Now we pre-multiply and post-multiply by the positive definite matrix $H^{-1 / 2}$ (whose existence is proved in (14) below):

$$
H^{1 / 2} P H^{-1 / 2}=-H^{-1 / 2} P^{*} H^{1 / 2}=-\left(H^{1 / 2} P H^{-1 / 2}\right)^{*}
$$

This skew-Hermitian matrix can be diagonalized by a unitary transformation :

$$
U(\xi) H^{1 / 2}(\xi) P(\xi) H^{-1 / 2}(\xi) U^{-1}(\xi)=D(\xi) .
$$

It remains to show that $S(\xi)=U(\xi) H^{1 / 2}(\xi)$ satisfies $|S|\left|S^{-1}\right| \leqslant$ constant. In fact, since $U$ is unitary, we have only to estimate $H^{1 / 2}$ and $H^{-1 / 2}$. For the latter,

$$
|v|^{2}=\left|\sum E_{j} v\right|^{2} \leqslant\left(\sum\left|E_{j} v\right|\right)^{2} \leqslant m \sum\left|E_{j} v\right|^{2}=\left|H^{1 / 2} v\right|^{2} .
$$

Therefore $\left|H^{-1 / 2}\right| \leqslant 1$.

We claim that each $E_{k}(\xi)$ is bounded by the constant in III. For this we set $z=\varepsilon+\lambda_{k}(\xi)$ and let $\varepsilon \rightarrow 0_{+}$:

(15) constant $\geqslant \operatorname{Re} z\left|(z-P(\xi))^{-1}\right|=\varepsilon\left|\sum\left(\varepsilon+\lambda_{k}-\lambda_{j}\right)^{-1} E_{j}\right|$

$$
\rightarrow\left|E_{k}\right| \text {. }
$$

This bound on each $\left|E_{k}\right|$ yields

$$
\begin{aligned}
\left|H^{1 / 2}(\xi) v\right|^{2}= & m \sum\left|E_{j}(\xi) v\right|^{2} \leqslant m^{2} \text { constant }^{2}|v|^{2} \\
& \left|H^{1 / 2}\right| \leqslant m \text { constant. }
\end{aligned}
$$

Thus I holds, with $|S|\left|S^{-1}\right|=\left|H^{1 / 2}\right|\left|H^{-1 / 2}\right|$ bounded by the order $m$ times the constant in III.

We point out a slight variant of III which will be useful later. It is apparently weaker, but actually equivalent.

III' $:^{\prime} \operatorname{Re} z\left|(z-P(\xi))^{-1}\right| \leqslant$ constant for $\operatorname{Re} z>0,|z| \leqslant 2|P(\xi)|$. $\mathrm{II} \Rightarrow \mathrm{III}^{\prime}$ is still all right, so we prove $\mathrm{III}^{\prime} \Rightarrow \mathrm{I}$. 
Certainly $P(\xi)$ can have no eigenvalue $\lambda$ in the semi-circle $\operatorname{Re} z>0$, $|z| \leqslant 2|P(\xi)|$. Nor can an eigenvalue be elsewhere in the halfplane $\operatorname{Re} z>0$, since $|\lambda|$ cannot exceed the norm $|P(\xi)|$. Replacing $\xi$ by $-\xi,(H)$ must hold. The basic estimate (15) also persists, since $z=\varepsilon+\lambda_{k}$ lies in the semi-circle as $\varepsilon \rightarrow 0_{+}$. Therefore the proof of I can go just as before.

3. THEOREM I: Each of the conditions I to III is necessary and sufficient for the first-order system (1) to be strongly hyperbolic.

$\mathrm{I} \Rightarrow$ strong hyperbolicity: Suppose I holds, and $\mu$ is an eigenvalue of $Q(\xi)=P(\xi)+B$. Then $\mu$ is also an eigenvalue of $S Q S^{-1}=$ $D+S B S^{-1}$, say with eigenvector $v$ :

$$
(D v, v)+\left(S B S^{-1} v, v\right)=\mu(v, v)
$$

Taking real parts, and using $I$, the first term drops out to leave (18) $\operatorname{Re} \mu \leqslant\left|S B S^{-1}\right| \leqslant$ constant $|B|$.

This holds for every $B$, so the system is strongly hyperbolic. This is essentially Petrowsky's proof $[7, \mathrm{p} .65]$; it might be interesting to point out how one could also prove

$\mathrm{II} \Rightarrow$ strong hyperbolicity: In [8], we carried out the following computation :

Lemma 1. If $\left|M^{n}\right| \leqslant C$ for $n \geqslant 0$, then $\left|(M+R)^{n}\right| \leqslant C e^{n C|R|}$.

To use II, we need to derive the exponential analogue:

Corollary. If $\left|e^{t P}\right| \leqslant C$ for $t \geqslant 0$, then $\left|e^{t: P+B)}\right| \leqslant C e^{t C|B|}$.

To prove the corollary, set $M=e^{\varepsilon P}$ and $R=e^{\varepsilon(P \div B)}-M$. Then $M$ satisfies the hypothesis of the lemma, so

$$
\left|(M+R)^{n}\right|=\left|e^{n \varepsilon(P+B)}\right| \leqslant C e^{n C|R|} \quad \text { for } n \geqslant 0 .
$$

Fixing $n \varepsilon=t, n R \rightarrow t B$ as $n \rightarrow \infty$, and the corollary follows.

Now we prove strong hyperbolicity. If $\mu$ is an eigenvalue of $Q=P+B$, then $e^{t \mu}$ is an eigenvalue of $e^{t(P+B)}$, and is therefore dominated by the norm:

(19) $e^{t \operatorname{Re} \mu}=\left|e^{t \mu}\right| \leqslant\left|e^{t(P+B)}\right| \leqslant C e^{t C|B|}$.

For large $t$, this requires that the exponents satisfy 
(20) $\operatorname{Re} \mu \leqslant C|B|$.

Thus we have strong hyperbolicity, and in fact with the same estimate for $\operatorname{Re} \mu$ as in Petrowsky's argument.

In the more delicate half of the argument, the proof that the three conditions are necessary, we look to condition III to play its part.

Strong hyperbolicity $\Rightarrow$ III: Suppose we have a strongly hyperbolic system (so that $(H)$ holds) for which III fails :

(21) $\operatorname{Re} z_{k}\left|\left(z_{k}-P\left(\xi^{k}\right)\right)^{-1}\right| \rightarrow \infty$

for some sequence $\xi^{k}$, with $\operatorname{Re} z_{k}>0$. These norms cannot get large without at least one of the $m^{2}$ entries, say the $(i, j)$ th, being responsible; taking a subsequence if necessary,

(22) $\operatorname{Re} z_{k}\left|\left(z_{k}-P\left(\xi^{k}\right)\right)^{-1}{ }_{i, j}\right| \rightarrow \infty$.

If $M(z, \xi)$ is the $(j, i)$ th minor of $z-P(\xi)$, then

(23) $(z-P(\xi))^{-1}{ }_{i, j}=\frac{(-1)^{i+j} M(z, \xi)}{\operatorname{det}(z-P(\xi))}$.

Let $B$ be a matrix whose only non-zero entry is $B_{j i}=$ $(-1)^{i+j} e^{i \phi}$. Then

(24) $R(z, \xi)=\operatorname{det}(z-Q(\xi))=\operatorname{det}(z-P(\xi))-e^{i \phi} M(z, \xi)$.

Our problem is to show that with a proper choice of $\phi$, the roots $\mu_{j}(\xi)$ of $R$ have unbounded real parts. Since this would contradict the hypothesis of strong hyperbolicity, we may then conclude that III cannot fail.

Fix $\xi$, and consider the behavior of $\mu_{j}(\beta \xi)$ as $\beta \rightarrow \infty$. Supposing for the moment that the eigenvalues $\lambda_{j}(\xi)$ are distinct, we expand into partial fractions :

(25) $\frac{M(z, \beta \xi)}{\operatorname{det}(z-P(\beta \xi))}=\frac{a_{1}(\beta \xi)}{z-\lambda_{1}(\beta \xi)}+\cdots+\frac{a_{m}(\beta \xi)}{z-\lambda_{m}(\beta \xi)}$.

$M$ is homogeneous in $z$ and $\beta$ of degree $m-1$, the determinant is of degree $m$, and the denominators $z-\lambda_{j}$ of degree one. Therefore the constants $a_{j}$ are homogeneous of degree zero: $a_{j}(\beta \xi)=$ 
$a_{j}(\xi)$. We want to prove that with the $\mu_{j}$ correctly ordered, (26)

$$
\mu_{j}(\beta \xi)-\lambda_{j}(\beta \xi) \rightarrow e^{i \phi} a_{j}(\beta \xi)=e^{i \phi} a_{j}(\xi) \text { as } \beta \rightarrow \infty \text {. }
$$

If $a_{j}=0$, this is easy ; $\lambda_{j}(\beta \xi)$ must be a root of $M$, or the left side of (25) would have a pole where the right side was regular. Therefore $\lambda_{j}$ is also a root of $R ; \lambda_{j}(\beta \xi) \equiv \mu_{j}(\beta \xi)$.

Assume $a_{j} \neq 0$ and take $\rho>0$ very small. We shall show that for large $\beta, R(z, \beta \xi)$ has exactly one root in the circle of radius $\rho$ about $\lambda_{j}(\beta \xi)+e^{i \phi} a_{j}(\beta \xi)$; this proves (26). The argument is based on Rouché's theorem. For large $\beta$,

$$
S(z, \beta \xi)=\operatorname{det}(z-P(\beta \xi))\left(1-\frac{e^{i \phi} a_{j}(\beta \xi)}{z-\lambda_{j}(\beta \xi)}\right)
$$

has exactly one root in the circle, at its center. So we have only to check that on the circle, $|R-S|<|S|$. Dividing by the determinant, this is the same as

$$
\left|\sum_{k \neq j} \frac{e^{i \phi} a_{k}(\beta \xi)}{z-\lambda_{k}(\beta \xi)}\right|<\left|1-\frac{e^{i \phi} a_{j}(\beta \xi)}{z-\lambda_{j}(\beta \xi)}\right| .
$$

The left side approaches zero, uniformly on the circle, as $\beta \rightarrow \infty$. The right side is

$$
\left|\frac{\rho e^{i \theta}}{e^{i \phi} a_{j}(\beta \xi)+\rho e^{i \theta}}\right| \geqslant \frac{\rho}{\left|a_{j}\right|-\rho}=\text { constant . }
$$

Therefore Rouché's theorem applies, and (26) holds.

The situation is not much changed when our fixed $\xi$ gives some coincident eigenvalues $\lambda_{j}$, provided there are still only linear factors in the partial fraction expansion of $M / \operatorname{det}(z-P)$. This occurs when every $p$ - fold root $\lambda_{j}$ of the denominator is at least a $p-1$ fold root of $M$. Suppose this is the case, and $m-n$ linear factors appear in the expansion. Then $\mu_{j}=\lambda_{j}$ for $n$ roots and (26) holds, by Rouché's theorem, for the other $m-n$.

We show now how (26), if it holds for every $\xi^{k}$, leads immediately to our theorem. Then we return to deal with the remaining possibility that some higher power $\left(z-\lambda_{j}\left(\xi^{k}\right)\right)^{g}, g>1$, appears in a partial fraction expansion.

The numbers $\left|a_{j}\left(\xi^{k}\right)\right|$ are not bounded by any constant $C$, 
since if they were, we would have

$$
\frac{\operatorname{Re} z_{k}\left|M\left(z_{k}, \xi^{k}\right)\right|}{\mid \operatorname{det}\left(z_{k}-P\left(\xi^{k}\right) \mid\right.} \leqslant \sum \frac{C \operatorname{Re} z_{k}}{\left|z_{k}-\lambda_{j}\left(\xi^{k}\right)\right|} \leqslant m C,
$$

contradicting (22-23). Therefore we may choose $\phi$ so that the numbers $\operatorname{Re}\left(e^{i \phi} a_{j}\left(\xi^{k}\right)\right)$ are unbounded above. According to (26),

$$
\operatorname{Re}\left(\mu_{j}\left(\beta \xi^{k}\right)\right) \rightarrow \operatorname{Re}\left(e^{i \phi} a_{j}\left(\xi^{k}\right)\right) \text { as } \beta \rightarrow \infty .
$$

Thus the roots of $R$ have unbounded real parts, as we intended to show.

Suppose finally that for some $\xi=\xi^{k}$, one of the partial fractions looks like

$$
\text { (27) } \frac{a_{j}(z, \beta \xi)}{\left(z-\lambda_{j}(\beta \xi)\right)^{g}}, g>1 \text {. }
$$

In this case $a_{j}$ is a homogeneous polynomial of degree $g-1$, and we may assume that (27) is irreducible: $a_{j}\left(\lambda_{j}(\xi), \xi\right)=c \neq 0$. Appealing one last time to Rouché's theorem, $g$ of the $\mu_{j}(\beta \xi)$ approach, as $\beta \rightarrow \infty$, the roots of

$$
\left(z-\lambda_{j}(\beta \xi)\right)^{g}=e^{i \dagger} a_{j}(z, \beta \xi) .
$$

It is not hard to show that these roots are

$$
\beta \lambda_{j}(\xi)+\omega_{k} \beta^{g-1 / g}\left(c e^{i \phi}\right)^{1 / g}+0\left(\beta^{\gamma}\right), \gamma<\frac{g-1}{g},
$$

where the $\omega_{k}$ are the $g$ th roots of unity. Take $\omega_{k}=1$ and choose $\phi$ so that $c e^{i \phi}=|c|$. Then the corresponding $\operatorname{Re}\left(\mu_{j}(\beta \xi)\right)$ grows like $\beta^{g-1 / g}|c|^{1 / g} \rightarrow \infty$. Thus hyperbolicity fails again, and the theorem is proved in this case without looking beyond the ray $\beta \xi$; this can happen only if $P(\xi)$ is not diagonalizable, as in the example (4-5). The earlier argument, with linear factors, covers the case when the $P(\xi)$ are all diagonalizable, but not uniformly so.

4. In this section we extend the discussion to a Kowalevsky system of arbitrary order, with unknowns $u_{1}(x, t), \cdots, u_{m}(x, t)$ :

(28) $\left(-\frac{\partial}{\partial t}\right)^{n_{j}} u_{j}=\sum_{k=1}^{m} \sum_{\alpha} A_{j k}^{\alpha}\left(\frac{\partial}{\partial t}\right)^{\alpha_{10}}\left(\frac{\partial}{\partial x_{1}}\right)^{\alpha_{1}} \cdots\left(\frac{\partial}{\partial x_{d}}\right)^{\alpha_{d}} u_{k}, \quad 1 \leqslant j \leqslant m$. 
The inner sum extends over derivatives of order $|\alpha|=\sum_{0}^{d} \alpha_{j} \leqslant n_{k}$, with $\alpha_{0}<n_{k}$. The derivatives $(\partial / \partial t)^{n} u_{j}, 0 \leqslant n<n_{j}$, are prescribed at $t=0$.

A Fourier transformation yields

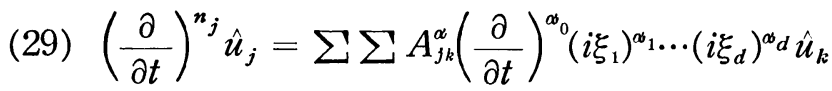

$$
=\sum_{k=1}^{m} Q_{j_{k}}^{\prime}\left(\frac{\partial}{\partial t}, \xi\right) \hat{u}_{k} \text {. }
$$

The derivatives of order $|\alpha|=n_{k}$ comprise the principal part; accordingly we split

$$
Q_{j k}^{\prime}=P_{j k}^{\prime}+B_{j k}^{\prime},
$$

where $P_{j_{k}}^{\prime}(\partial / \partial t, \xi)$ is a homogeneous polynomial in $\partial / \partial t, \xi_{1}, \cdots, \xi_{d}$ of degree $n_{k}$, and $B_{j_{k}}^{\prime}$ is of lower degree. Corresponding to the left side of (29), we introduce the diagonal matrix $E(z)$ which has $E_{j j}=z^{n_{j}}$. Then the Petrowsky-Gårding condition, generalizing (3), reads :

The system (28) is hyperbolic if $\operatorname{Re} \mu_{j}(\xi) \leqslant$ constant, where the $\mu_{j}$ are the roots of $\operatorname{det}\left(E(z)-Q^{\prime}(z, \xi)\right)$.

Strong hyperbolicity means, of course, hyperbolicity for every choice of $B^{\prime}$. The choice $B^{\prime}=0$ again yields

$(H)$ : If $(28)$ is strongly hyperbolic, then the roots $\lambda_{j}(\xi)$ of $\operatorname{det}\left(E(z)-P^{\prime}(z, \xi)\right)$, which is a homogeneous polynomial of degree $N=\sum n_{j}$, are all imaginary.

Naturally we want to study (29) by reducing it to a firstorder system. It turns out that a reduction to a differential system (1) is less useful than turning to a pseudo-differential system; in other words, we shall arrive at a non-polynomial symbol $Q(\xi)$. Corresponding to the unknown $\hat{u}_{j}$ we introduce $n_{j}$ new unknowns

$$
\hat{v}_{j, r}(t, \xi)=|\xi|^{n_{j-1-r}}\left(\frac{\partial}{\partial t}\right)^{r} \hat{u}_{j}(t, \xi), \quad 0 \leqslant r \leqslant n_{j}-1
$$

Then we may rewrite (28) as 


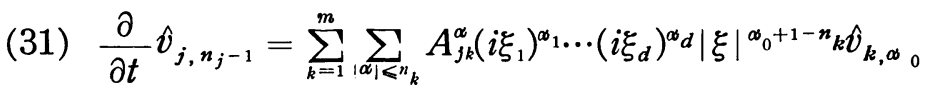

Notice that derivatives of order $|\alpha|=n_{k}-g$ yield terms which are homogeneous in $\xi$ of degree $1-g$. There are also the usual auxiliary equations

$$
\frac{\partial}{\partial t} \hat{v}_{j, r}=|\xi| \hat{v}_{j, r+1}, 1 \leqslant j \leqslant m, 0 \leqslant r \leqslant n_{j}-2
$$

Suppose we combine the components $\hat{v}_{j, r}$ into an unknown vector $\hat{v}$ of order $N=\sum n_{j}$. Then (31-32) can be written as a pseudodifferential system

(33) $\frac{\partial \hat{v}}{\partial t}=Q(\xi) \hat{v}=(P(\xi)+B(\xi)) \hat{v}$.

The $N \times N$ matrix $P$, corresponding to the principal part of (31) and to the equations (32), is homogeneous of degree one. We must distinguish between its $m$ main rows, which arise from (31), and the $N-m$ auxiliary rows, which have a single non-zero entry $|\xi|$. The lower-order term $B(\xi)$ is bounded as $|\xi| \rightarrow \infty$, but will be unbounded as $|\xi| \rightarrow 0$ if there are any derivatives of order $|\alpha|<n_{k}-1$.

To complete the book-keeping, we establish the relationship between $Q^{\prime}$ and the new coefficients $Q$. Let us introduce the $N \times N$ matrix

$$
Y(z, \xi)=\left(\begin{array}{cc}
E(z)-Q^{\prime} & 0 \\
0 & I_{N-m}
\end{array}\right) .
$$

LEMMA 2. a) There exist matrices $L$ and $R$, with entries which are polynomials in $z, \xi$, and $|\xi|^{-1}$, such that $L Y R=z-Q(\xi)$ and det $L R=1$.

(b) $\operatorname{det}\left(E(z)-Q^{\prime}(z, \xi)\right)=\operatorname{det} Y=\operatorname{det}(z-Q(\xi))$, with roots $\mu_{j}(\xi)$, and $\operatorname{det}\left(E(z)-P^{\prime}(z, \xi)\right)=\operatorname{det}(z-P(\xi))$, with roots $\lambda_{j}(\xi) . \quad(c)$ Every minor (of order $N-1$ ) of $z-P$ is a linear combination-with coefficients which are polynomials in $z, \xi$, and $|\xi|^{-1}$, and homogeneous of degree zero in $z$ and $\xi$-of the main minors of $z-P$, i.e., of those minors formed by omitting a main row and an arbitrary column. 
We shall be very brief about the proof, since this result was drawn from Lemma 1-2 in [2]. Every factor $|\xi|$ in the entries of $Q$ is replaced by 1 after the proper similarity transformation $T$, namely the one which changes the unknowns to

$$
\hat{v}_{j, r}^{*}=|\xi|^{r+1-n_{j}} \hat{v}_{j, r}=\left(\frac{\partial}{\partial t}\right)^{r} \hat{u}_{j} .
$$

( $T$ is a diagonal matrix constructed from powers of $|\xi|$. ) Then the matrices $T(z-Q) T^{-1}$ and $Y$ are equivalent under elementary row and column operations. Using the off-diagonal -1 's in the auxiliary rows as pivots, we obtain all the zero entries of $Y$; these operations involve polynomials in $z$ and $\xi$, and have unit determinant. With appropriate permutations and a change of sign, the -1 's become $I_{N-m}$. Together with the similarity, all these operations make up $L$ and $R$.

The first equality in b) is obvious from a), and the second follows by comparing the terms of highest order. A useful diagram for step a), and the proof of c), appear in [2].

5. Theorem II. Each of the conditions I to $I I I$ (as well as $I I I^{\prime}$ ) is necessary and sufficient for the Kowalevsky system (28) to be strongly hyperbolic.

Proof. I $\Rightarrow$ strong hyperbolicity. For any choice of lowerorder terms, $|B(\xi)| \leqslant$ constant for $|\xi| \leqslant 1$. We can therefore apply, in this range, the same argument as in Theorem I. On the other hand, $\operatorname{det}\left(E(z)-Q^{\prime}(z, \xi)\right)$ is a monic polynomial with coefficients bounded in the range $|\xi| \leqslant 1$. Therefore $\operatorname{Re} \mu_{j}(\xi) \leqslant$ constant also for $|\xi| \leqslant 1$.

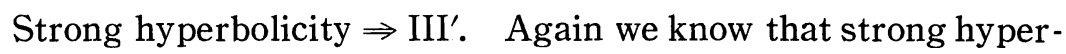

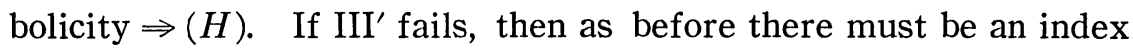
$(I, J)$ such that

$$
\operatorname{Re} z_{k}\left|\left(z_{k}-P\left(\xi^{k}\right)\right)^{-1}{ }_{I, J}\right| \rightarrow \infty,
$$

where $\operatorname{Re} z_{k}>0$ and $\left|z_{k}\right| \leqslant 2\left|P\left(\xi^{k}\right)\right|$. In other words, the corresponding minor satisfies

(34) $\frac{\operatorname{Re} z_{k}\left|M_{J I}\left(z_{k}, \xi^{k}\right)\right|}{\mid \operatorname{det}\left(z_{k}-P\left(\xi^{k}\right) \mid\right.} \rightarrow \infty$. 
We claim that this holds for at least one of the main minors. A polynomial in $z$ which is also homogeneous of order zero in $z$ and $\xi$ together is uniformly bounded in the range $|z| \leqslant 2|P(\xi)|$; in fact a typical term in our polynomials can be estimated by

$$
\left|\frac{\text { constant } z^{n} \xi_{1}^{\alpha} \ldots \xi_{d}^{\alpha}}{|\xi|^{n+\left|\alpha_{d}\right|}}\right| \leqslant \text { constant }\left(2 \sup _{|\xi|=1}|P(\xi)|\right)^{n} .
$$

According to Lemma III c), $M_{J I}$ is either a main minor or a combination of the main minors with such polynomials as coefficients. Since the coefficients are bounded, (34) cannot hold for $M_{J I}$ without holding also for a main minor $M_{j i}$.

Now we introduce an appropriate lower-order term into our system, namely one which will produce a single non-zero entry $B(\xi)_{j i}=b(\xi)$. This we can do precisely because the $j$ th row is a main row; the auxiliary rows correspond to the fixed equations (32) and cannot be altered. Suppose the column index $i$ corresponds to terms in (31) multiplying $\hat{v}_{k, r}$. Then we want our particular lower-order term to have $|\alpha|=n_{k}-1, \alpha_{0}=r$, and constant coefficient $(-1)^{i+j} e^{i \downarrow}$; this will produce

$$
b(\xi)=\frac{(-1)^{i+j} e^{i \phi}\left(i \xi_{1}\right)^{\alpha_{1} \ldots \cdot\left(i \xi_{d}\right)^{\alpha_{d} t}}}{|\xi|^{\omega_{1} \ldots \cdot|\xi|^{\alpha_{d}}}}
$$

We emphasize that although $B$ is homogeneous of degree zero, it is not a constant matrix (unless $n_{k}=1$, as was always the case in Theorem I). This difficulty is overcome by taking a subsequence, if necessary, so that the vectors $\xi^{k} /\left|\xi^{k}\right|$ converge to a unit vector $\xi^{\infty}$. Then copying the argument in Theorem I (assuming linear factors in the partial fractions) we have

(35) $\operatorname{Re} \mu_{j}\left(\beta \xi^{k}\right) \rightarrow \operatorname{Re}\left(b\left(\xi^{k}\right) a_{j}\left(\xi^{k}\right)\right)$ as $\beta \rightarrow \infty$;

$\mu_{j}$ are the eigenvalues of $Q=P+B$, and the $a_{j}\left(\xi^{k}\right)$ are unbounded.

Choose $\phi$ so that for some subsequence $\eta^{k}$ of the $\xi^{k}$, and for appropriate $j=j(k)$, we have

$$
\left|b\left(\xi^{\infty}\right) a_{j}\left(\eta^{k}\right)\right| \rightarrow \infty, \quad \mid \arg \left(b\left(\xi^{\infty}\right) a_{j}\left(\eta^{k}\right) \mid<\pi / 4 .\right.
$$

Multiplying by $b\left(\eta^{k}\right) / b\left(\xi^{\infty}\right)$, which approaches 1 , neither the modulus nor the argument is much changed. Therefore 
(36) $\operatorname{Re}\left(b\left(\eta^{k}\right) a_{j}\left(\eta^{k}\right)\right) \rightarrow \infty$.

Comparing this with (35), $\operatorname{Re} \mu_{j}\left(\beta \xi^{k}\right)$ cannot be bounded above, and the lower-order term which produced $B$ has destroyed hyperbolicity.

When there is a non-linear factor (27), for some $\xi=\xi^{k}$, one chooses $\phi$ so that $c b\left(\xi^{k}\right)>0$. Then $\operatorname{Re} \mu_{j}\left(\beta \xi^{k}\right) \rightarrow \infty$ as $\beta \rightarrow \infty$, as before, and hyperbolicity is destroyed here too.

We conclude that $\mathrm{III}^{\prime}$, and therefore, I, II, and III, cannot fail.

6. The reduced Kowalevsky system $\frac{\partial \hat{v}}{\partial t}=Q(\xi) \hat{v}$ has the obvious solution

$$
\hat{v}(\xi, t)=e^{t Q(\xi)} \hat{v}(\xi, 0) .
$$

Unfortunately, $e^{t Q}=e^{t(P+B)}$ may be unbounded as $|\xi| \rightarrow 0$, since we cannot guarantee that $B$ is bounded. In other words, while $\hat{v}$ was an appropriate choice for the analysis of strong hyperbolicity, it may not depend continuously on the data at $t=0$. Since the difficulty arises when $\xi$ is small, we introduce instead the unknowns

$$
\hat{w}_{j, r}(t, \xi)=\left(|\xi|^{2}+1\right)^{\left(n_{\left.j^{-1}-r\right) / 2}\right.}\left(\frac{\partial}{\partial t}\right)^{r} \hat{u}_{j}(t, \xi), 0 \leqslant r \leqslant n_{j}-1 .
$$

This leads to a corresponding system of order $N$ :

$$
\frac{\partial \hat{w}}{\partial t}=\widetilde{Q}(\xi) \hat{w}=(\widetilde{P}(\xi)+\widetilde{B}(\xi)) \hat{w}
$$

The part $\widetilde{B}$ arising from derivatives of order $|\alpha|<n_{k}$ in (28) is bounded : $|\widetilde{B}(\xi)| \leqslant$ constant.

$\widetilde{P}$ is no longer homogeneous; it can be formed from our previous $P$ by replacing every factor $|\xi|$ by $\left(|\xi|^{2}+1\right)^{1 / 2}$. We need two inequalities to check that this change leaves $\mid \tilde{P}(\xi)-$ $P(\xi) \mid \leqslant$ constant. On the auxiliary rows,

$$
\left|\left(|\xi|^{2}+1\right)^{1 / 2}-\right| \xi|| \leqslant 1 \text {. }
$$

On the main rows, the numerator of a typical term is a polynomial in $\xi$, say of degree $k+1$, and then 


$$
\left|P_{k+1}(\xi)\left(|\xi|^{-k}-\left(|\xi|^{2}+1\right)^{-k / 2}\right)\right| \leqslant \text { constant. }
$$

Thus $\widetilde{Q}-P=\widetilde{B}+(\widetilde{P}-P)$ is bounded uniformly in $\xi$.

Now we apply the corollary to Lemma I. If the Kowalevsky system (28) is strongly hyperbolic, then II holds, for some constant $C$. Therefore

$$
\left|e^{t \tilde{Q}(\xi)}\right|=\left|e^{t P+t[\tilde{Q}-P]}\right| \leqslant C e^{\rho t} .
$$

The constants $C$ and $\rho=C \sup _{\xi}|\widetilde{Q}-P|$ are independent of $\xi$.

Conversely, suppose $\left|e^{t \widetilde{Q}(\xi)}\right| \leqslant C^{\prime} e^{\rho^{\prime t}}$ for some $C^{\prime}$ and $\rho^{\prime}$. Then $\left|e^{t\left(\tilde{Q}-\rho^{\prime} I\right)}\right| \leqslant C^{\prime}$ and the corollary gives $\left|e^{t P}\right| \leqslant C^{\prime} e^{\rho^{\prime \prime t}}$. But $P$ is homogeneous, so for any $t$ and $\xi$,

$$
\left|e^{t P(\xi)}\right|=\left|e^{\varepsilon P(t \xi / \varepsilon)}\right| \leqslant C^{\prime} e^{\rho / \ell} \rightarrow C^{\prime}, \text { as } \varepsilon \rightarrow 0 .
$$

Thus II holds, and the system is strongly hyperbolic. We have proved

THEOREM III. The Kowalevsky system is strongly hyperbolic if and only if, for the new variables $\hat{w}_{j, r}$ defined by (37), there are constants $C$ and $\rho$ such that $t \geqslant 0$ and all $\xi$,

$$
\left|\hat{w}_{j r}(\xi, t)\right|=\left|e^{t \tilde{Q}(\xi)} \hat{w}_{j, r}(\xi, 0)\right| \leqslant C e^{\rho t}\left|\hat{w}_{j, r}(\xi, 0)\right|
$$

Corollary. To the Kowalevsky system (28) we associate the norm

$$
\begin{aligned}
\|u(t)\|^{2} & =\sum_{j=1}^{m} \sum_{\mid \alpha_{\mid<n_{j}}} \int_{R^{d}}\left|\left(\frac{\partial}{\partial t}\right)^{\infty_{0}}\left(\frac{\partial}{\partial x_{1}}\right)^{\alpha_{1}} \cdots\left(\frac{\partial}{\partial x_{d}}\right)^{\alpha_{d} t} u_{j}\right|^{2} d x \\
& =\sum_{j} \sum_{\mid \alpha_{\mid<n_{j}}}\left\|D^{\infty} u_{j}\right\|_{L_{2}}^{2}
\end{aligned}
$$

Then the system is strongly hyperbolic if and only if there are constants $C^{\prime}$ and $\rho^{\prime}$ such that for any initial data and any $t \geqslant 0$,

$$
\|u(t)\| \leqslant C^{\prime} e^{\rho / t}\|u(0)\| \text {. }
$$

Proof: The theorem gives this result immediately in the norm

$$
\begin{aligned}
\|u(t)\|_{0}^{2} & =\sum_{j, r} \int\left|\hat{w}_{j, r}(\xi, t)\right|^{2} d \xi . \\
& =\sum_{j, r} \int\left(|\xi|^{2}+1\right)^{n_{j}-1-r}\left|\left(\frac{\partial}{\partial t}\right)^{r} \hat{u}_{j}\right|^{2} d \xi .
\end{aligned}
$$

By Parseval's Theorem 


$$
\int\left(|\xi|^{2}+1\right)|\hat{g}(\xi)|^{2} d \xi=\int\left(\left|\frac{\partial g}{\partial x_{1}}\right|^{2}+\cdots+\left|\frac{\partial g}{\partial x_{d}}\right|^{2}+|g|^{2}\right) d x .
$$

After $n_{j}-1-r$ applications, we find positive constants $b_{\alpha r}$ and $c_{a s}$ such that

$$
\begin{aligned}
\|u(t)\|_{0}^{2} & =\sum_{j, r} \sum_{\substack{\mid \alpha_{\mid<n_{j}} \\
\alpha_{0}=r}} b_{a r r}\left\|D^{\alpha} u_{j}(t)\right\|_{L_{2}}^{2} \\
& =\sum_{j} \sum_{\mid \alpha_{\mid<n_{j}}} c_{a b}\left\|D^{\alpha} u_{j}(t)\right\|_{L_{2}}^{2} .
\end{aligned}
$$

In short, the norms \|\|$_{0}$ and \|\| are equivalent. Therefore the system is well-posed also in the latter norm.

7. Our only problem in extending these results to partial difference equations is to get all the analogies right. We shall consider systems of the form

(39) $u(x, t+k, k)=\sum c_{j}(k) u(x+j h, t, k)$,

where $u$ is an unknown $m$-vector, prescribed at $t=0$, and the matrix coefficients $c_{j}$ are polynomials in $k$. As usual $x=\left(x_{1}, \cdots, x_{d}\right)$ and $j=\left(j_{1}, \cdots, j_{d}\right)$. We are interested in the behavior of $u$ as $k$ and $h=h(k)$ approach zero. Taking Fourier transforms, and putting $h \xi=\theta$,

$$
\begin{aligned}
\hat{u}(\xi, t+k, k) & =\left(\sum c_{j}(k) e^{i j \cdot h \xi}\right) \hat{u}(\xi, t, k) \\
& =\left(\sum c_{j}(k) e^{i j \cdot \theta}\right) \hat{u}=Q(\theta, k) \hat{u} .
\end{aligned}
$$

We call the systen reversible (in time) when all the eigenvalues $\mu_{j}(\theta, k)$ of the amplification matrix $Q$ satisfy

(41) $1-C k \leqslant\left|\mu_{j}(\theta, k)\right| \leqslant 1+C k$,

for some fixed $C$. (This is to be compared with the condition (3) for hyperbolicity, which involved only a one-sided inequality $\operatorname{Re} \mu \leqslant C$. The homogeneity of $P$ then implies the other side $\operatorname{Re} \mu \geqslant-C^{\prime}$; here we have no such weapon.)

The system is strongly reversible if for every term bounded by $|B(\theta, k)|=0(k)$, the system whose amplification matrix is $Q+B$ remains reversible. The principal part of $Q$ is made up of the terms which are independent of $k$ : 


$$
P(\theta)=\sum c_{j}(0) e^{i j \cdot \theta} .
$$

Obviously there is the necessary condition

$\left(H^{\prime}\right)$ : If (39) is strongly reversible, then all the eigenvalues $\lambda_{j}(\theta)$ of $P$ must have $\left|\lambda_{j}\right|=1$.

The theorem which follows is completely analogous to Theorem I, so perhaps we may omit its proof.

Theorem IV: Each of the following conditions $I^{\prime}$ to $I I I^{\prime}$ is necessary and sufficient for the system (39) to be strongly reversible:

$I^{\prime}$. $\left(H^{\prime}\right)$ holds, and the matrices $P(\theta)$ are uniformly diagonalizable.

II'. $\left|P^{n}(\theta)\right| \leqslant$ constant, for $n \geqslant 0$ and real $\theta$.

III'. $(|z|-1)\left|(z-P(\theta))^{-1}\right| \leqslant$ constant, for $|z|>1$ and real $\theta$.

In proving that strong reversibility fails whenever III' does, we can ensure that it is the second inequality in (41) which is violated. This means, as we mentioned in the introduction, that weak stability is always vulnerable to $O(k)$ perturbations.

To complete the discussion, we observe that $\mathrm{II}^{\prime}$ is equivalent to stability in the Lax-Richtmyer sense; according to Lemma 1, $\left|P^{n}(\theta)\right| \leqslant C$ if and only if

$$
\left|Q^{n}\right|=\left|(P+0(k))^{n}\right| \leqslant C e^{n C(1)} \leqslant \text { constant for } n k \leqslant T .
$$

Thus, for reversible systems, Lax-Richtmyer stability is equivalent to strong reversibility.

\section{Appendix}

As we remarked in the introduction, each of the following hypotheses implies the next:

a) For some $T$, the matrices $T A_{j} T^{-1}$ are all Hermitian

b) The system (1) is strongly hyperbolic

c) Every real combination $\sum \xi_{j} A_{j}$ is similar to a real diagonal matrix.

The implications become obvious if we replace strong hyperbolicity by the equivalent condition I of our first Theorem. For 
systems in two unknowns, these three hypotheses are actually equivalent; the proof is depressingly straightforward.

THEOREM V. Given $2 \times 2$ matrices such that every real combination $A_{1} \xi_{1}+\cdots+A_{d} \xi_{d}$ is similar to a real diagonal matrix. Then for some $T$, the matrices $T A_{j} T^{-1}$ are all Hermitian.

Proof. First we diagonalize $A_{1} ; A_{1}^{\prime}=S A_{1} S^{-1}=\left(\begin{array}{cc}a_{1} & 0 \\ 0 & a_{2}\end{array}\right)$, where $a_{1}$ and $a_{2}$ are real. We assume $a_{1} \neq a_{2}$, since otherwise $A_{1}$ is a real multiple of the identity and can be ignored.

Suppose $A_{2}{ }^{\prime}=S A_{2} S^{-1}=\left(\begin{array}{ll}b_{1} & b_{3} \\ b_{4} & b_{2}\end{array}\right)$. If either $b_{3}$ or $b_{4}$ is zero, so is the other, since otherwise some combination of $A_{1}{ }^{\prime}$ and $A_{2}{ }^{\prime}$ is not similar to a real diagonal matrix. If both $b_{3}$ and $b_{4}$ vanish, then $A_{2}$ can be ignored, since whatever diagonalizes $A_{1}$ also diagonalizes $A_{2}$. Now we show that $b_{1}$ and $b_{2}$ are real, and that $b_{3} b_{4} \neq 0$ implies $b_{3} b_{4}>0$. The characteristic equation of $A_{1}^{\prime} \xi+A_{1}^{\prime} \eta$ is

$$
\left(\lambda-a_{1} \xi-b_{1} \eta\right)\left(\lambda-a_{2} \xi-b_{2} \eta\right)-b_{3} b_{4} \eta^{2}=0
$$

and this has real roots if and only if its discriminant satisfies

$$
\left[\left(a_{1}-a_{2}\right) \xi+\left(b_{1}-b_{2}\right) \eta\right]^{2}+4 b_{3} b_{4} \eta^{2} \geqslant 0 .
$$

This quadratic form is non-negative for real $\xi, \eta$ if and only if

(i) the coefficients are real; in particular, the coefficient $\left(a_{1}-a_{2}\right)\left(b_{1}-b_{2}\right)$ of $\xi \eta$ must be real. Thus $b_{1}-b_{2}$ is real and since the trace $b_{1}+b_{2}$ is real (the sum of the eigenvalues of $A_{2}$ ), we conclude that $b_{1}$ and $b_{2}$ are real.

(ii) the determinant of the associated matrix is $\geqslant 0$, i.e.,

$$
\left(a_{1}-a_{2}\right)^{2}\left[\left(b_{1}-b_{2}\right)^{2}+4 b_{3} b_{4}\right]-\left(a_{1}-a_{2}\right)^{2}\left(b_{1}-b_{2}\right)^{2} \geqslant 0
$$

which gives $b_{3} b_{4}>0$.

Now with $D=\left(\begin{array}{ll}1 & 0 \\ 0 & d\end{array}\right), d=b_{3}\left(b_{3} b_{4}\right)^{-1 / 2}$, we set $T=D S$, $A_{j}{ }^{\prime \prime}=T A_{j} T^{-1}$, and find

$$
A_{1}^{\prime \prime}=\left(\begin{array}{ll}
a_{1} & 0 \\
0 & a_{2}
\end{array}\right), \quad A_{2}^{\prime \prime}=\left(\begin{array}{ll}
b_{1} & b \\
b & b_{2}
\end{array}\right), \quad A_{3}^{\prime \prime}=\left(\begin{array}{ll}
c_{1} & c_{3} \\
c_{4} & c_{2}
\end{array}\right) .
$$


The entries of $A_{1}^{\prime \prime}$ and $A_{2}^{\prime \prime}$ are real, and we have already made $a_{1} \neq a_{2}$ and $b=\left(b_{3} b_{4}\right)^{1 / 2} \neq 0$ to rule out trivial cases.

We want to show that $A_{3}^{\prime \prime}$ (and similarly every $A_{j}^{\prime \prime}$ ) is Hermitian. Applying the second paragraph of the proof to $A_{3}^{\prime \prime}$ instead of $A_{2}^{\prime}$, we know that $c_{1}$ and $c_{2}$ are real, and that either $c_{3}$ and $c_{4}$ both vanish (in which case $A_{3}{ }^{\prime \prime}$ is Hermitian) or $c_{3} c_{4}>0$.

Now every real combination $A_{1}^{\prime \prime} \xi+A_{2}{ }^{\prime \prime} \eta+A_{3}{ }^{\prime \prime} \mu+I \nu$ has only real eigenvalues. For each $\eta, \mu$ we can choose $\xi, \nu$ to cancel the diagonal elements; thus

$$
\left(\begin{array}{cc}
0 & b \eta+c_{3} \mu \\
b \eta+c_{4} \mu & 0
\end{array}\right)
$$

has real eigenvalues for every $\eta, \mu$. Since the trace is zero, the determinant is not positive, i.e.,

$$
\left(b \eta+c_{3} \mu\right)\left(b \eta+c_{4} \mu\right) \geqslant 0
$$

The coefficient of the cross-product term is real, so $c_{3}+c_{4}$ is real. Also the determinant of the associated matrix is non-negative, so

$$
\begin{aligned}
& b^{2} c_{3} c_{4} \geqslant\left(\frac{b\left(c_{3}+c_{4}\right)}{2}\right)^{2} \\
& b^{2}\left(c_{3}-c_{4}\right)^{2} \leqslant 0
\end{aligned}
$$

and $c_{3}-c_{4}$ is pure imaginary. Thus $c_{3}=\overline{c_{4}}$, completing the proof.

It is natural to enquire whether hyperbolicity, as well as strong hyperbolicity, has a simple description for matrices $A_{j}$ and $B$ of order two. Certainly hyperbolicity is implied either by strong hyperbolicity or by the alternative hypothesis

$\mathrm{a}^{\prime}$ ) For some $T$, the matrices $T A_{j} T^{-1}$ and $T B T^{-1}$ are all upper triangular, and the $A_{j}$ have real eigenvalues.

The proof of the converse is again perfectly direct.

THEOREM VI. A first-order system in two unknowns is hyperbolic if and only if either a) or $a^{\prime}$ ) is satisfied.

Proof. Suppose the system is hyperbolic, but a) is not satisfied. According to Theorem $\mathrm{V}$, some combination $C=\sum A_{j} \xi_{j}$ is not similar to a real diagonal matrix. Since it is well-known that the principal part of a hyperbolic system is hyperbolic (in 
other words, $(H)$ is necessary for the hyperbolicity of (1)) the eigenvalues $\lambda$ of $C$ are real, and it must be the diagonalizability which fails. Suppose $T$ puts $C$ into Jordan form:

$$
C^{\prime}=T C T^{-1}=\left(\begin{array}{cc}
\lambda & 1 \\
0 & \lambda
\end{array}\right) \text { and } A_{j}^{\prime}=T A_{j} T^{-1}=\left(\begin{array}{ll}
a_{j} & c_{j} \\
d_{j} & b_{j}
\end{array}\right) \text {, say. }
$$

The problem is to show that each $d_{j}$ vanishes. By the hyperbolicity of the principal part, every real combination $A_{j}{ }^{\prime}+\alpha C^{\prime}$ has real eigenvalues. Since $\lambda$ is real, this means that

$$
\left(\begin{array}{cc}
a_{j} & c_{j}+\alpha \\
d_{j} & b_{j}
\end{array}\right) \text { has only real eigenvalues. }
$$

The (quadratic) characteristic equation has real roots only if its discriminant satisfies

$$
\left(a_{j}-b_{j}\right)^{2}+4 d_{j}\left(c_{j}+\alpha\right) \geqslant 0 .
$$

This is possible for all real $\alpha$ only if $d_{j}=0$.

The hyperbolicity of (1) also requires the real parts of the eigenvalues of $i \alpha C+B$ to be bounded for $-\infty<\alpha<\infty$. But these are the eigenvalues of $i \alpha C^{\prime}+T B T^{-1}$, and it is easy to check that $T B T^{-1}$ is forced to be upper triangular. Thus $\mathrm{a}^{\prime}$ ) is satisfied, completing the argument.

We summarize very briefly the corresponding situation for a single second-order equation of Kowalevsky type. It is strongly hyperbolic if and only if a suitable transformation of the independent variables $\left(t, x_{1}, \cdots, x_{d}\right)$ produces the wave equation (with lower order terms) in $d$ space variables. An equation which is hyperbolic, but not strongly hyperbolic, yields the wave equation in fewer variables.

Friedrichs has pointed out an alternative reduction, to a firstorder symmetric hyperbolic system. We mention that the description in Courant-Hilbert II (p. 594) is not quite correct as it stands; the strongly hyperbolic equation $u_{t t}=u_{t x}$ would there become

$$
\left(\begin{array}{ll}
1 & 0 \\
0 & 0
\end{array}\right) v_{t}=\left(\begin{array}{ll}
1 & 0 \\
0 & 0
\end{array}\right) v_{x}
$$


and the coefficient of $v_{t}$ is singular. The description given is valid once the terms in $u_{t x_{i}}$ have been removed, for example by a change of the time variable.

Massachusetts Institute of Technology.

\section{REFERENCES}

[1] Yamaguti, M. and Kasahara, K.: Sur le système hyperboliquc à coefficients constants, Proc. Japan Acad. Vol. 35 (1959) pp. 547-550.

[2] Kasahara, K. and Yamaguti, M. : Strongly hyperbolic system of linear partial differential equations with constant coefficients, Memoirs of College of Science, Univ. of Kyoto, Series A. Vol. XXXIII. Maths. No. 1, 1960.

[3] Kreiss, H. O.: Über die Stabilitätsdefinition für Differenzengleichungen die partielle Differentialgleichungen approximieren, B. I. T. 2 (1962) 153-181.

[ 4 ] Forsythe, G. E. and Wasow, W. R. : Finite-difference methods for partial differential equations, (John Wiley \& Sons, Inc., Publishers, 1960).

[5] Kreiss, H. O.: Über Matrizen die beschränkte Halbgruppen erzeugen, Math. Scand. 7 (1959) 71-80.

[6] Miller, J. and Strang, G. : Matrix theorems for partial differential and difference equations, Math. Scand. 18 (1966) pp. 113-133.

[7] Petrowsky, I. G. : Über das Cauchysche Problem für eine System linearer partieller Differentialgleichungen im Gebiete der nichtanalytischen Funktionen, Bull. Univ. d'Etat Moscou, Vol. 1, fasc. 7 (1938) pp. 1-74.

[8] Strang, G. : Accurate Partial Difference Methods, II. Non-Linear Problems, Num. Math. 6, 37-46 (1964).

[9] Lax, P. D.: Differential equations, difference equations, and matrix theory, Comm. Pure Appl. Math. 11 (1958) pp. 175-194.

[10] Motzkin, T. S., and Taussky, Olga : Pairs of matrices with property L, Trans. Amer. Math. Soc. 73 (1952) pp. 108-114. 